\title{
Diffusion and perfusion MR patterns of central nervous system lymphomas
}

\author{
Małgorzata Neska-Matuszewska ${ }^{\mathrm{A}-\mathrm{E}}$, Anna Zimny ${ }^{\mathrm{A}-\mathrm{C}, \mathrm{E}, \mathrm{F}}$, Joanna Bladowska ${ }^{\mathrm{A}, \mathrm{E}}$, Marek Sąsiadek ${ }^{\mathrm{E}, \mathrm{F}}$ \\ Department of General and Interventional Radiology and Neuroradiology, Wroclaw Medical University, Poland \\ A - research concept and design; B - collection and/or assembly of data; $C$ - data analysis and interpretation; \\ $D$ - writing the article; $E$ - critical revision of the article; $F$ - final approval of the article
}

\begin{abstract}
Małgorzata Neska-Matuszewska
Funding sources

None declared

\section{Conflict of interest}

None declared
\end{abstract}

Address for correspondence

E-mail: neskamatuszewska@gmail.com

Received on December 29, 2016

Reviewed on April 20, 2017

Accepted on May 19, 2017
DOI

10.17219/acem/73894

\section{Copyright}

Copyright by Author(s)

This is an article distributed under the terms of the

Creative Commons Attribution Non-Commercial License

(http://creativecommons.org/licenses/by-nc-nd/4.0/)

\section{Abstract}

Background. Central nervous system lymphomas (CNSLS) are rare tumors which may show variable appearance in standard magnetic resonance imaging (MRI) depending on their origin (primary or secondary) or patients' immunological status.

Objectives. The aim of the study was to analyze imaging patterns of different CNSLS, using diffusion-weighted imaging (DWI) and perfusion-weighted imaging (PWI).

Material and methods. Our material consisted of 16 CNSLS (14 primary, 2 secondary, 13 immunocompetent, 3 immunodeficient) which underwent magnetic resonance (MR) examinations including DWI and $T 2^{*}$ dynamic susceptibility contrast (DSC) perfusion (without a preload in 13 cases, with a preload in 3 subjects). In DWI, apparent diffusion coefficient (ADC), and in PWI, parameters of relative cerebral blood volume (rCBV), relative peak height (rPH) and relative percentage of signal recovery (rPSR) were analyzed within the entire tumor (mean values) and in regions with minimal diffusion $\left(A D C_{\text {min }}\right)$ and maximal perfusion values (rCBV max $_{1}, \mathrm{PH}_{\max ,} \mathrm{rPSR}$ max $)$.

Results. All CNSLs showed low values of $A D C_{\text {mean }}(0.70 \times 10-3), A D C_{\text {min }}(0.54 \times 10-3), r C B V_{\text {mean }}(0.80)$, $r C B V_{\text {max }}(1.27), r P H_{\text {mean }}(1.05), r P H_{\text {max }}(1.59)$, as well as high values of rPSR mean (1.99) and rPSR max $(2.41)$. There were no significant differences in rCBV $\mathrm{max}_{\text {, }}$ as well as in all ADC, rPH and rPSR values between primary and secondary CNSLs or between tumors in immunocompetent and immunodeficient patients. Dynamic susceptibility contrast PWI with a preload resulted in significantly higher rCBV, rPH and lower rPSR values.

Conclusions. Despite various MR appearances, both primary and secondary CNSLs in immunocompetent and immunodeficient patients show very typical patterns of restricted diffusion and hypoperfusion with signal intensity curves returning above the baseline. Dynamic susceptibility contrast perfusion without a preload is recommended.

Key words: central nervous system lymphomas, diffusion-weighted imaging, perfusion-weighted imaging, magnetic resonance imaging 


\section{Introduction}

Central nervous system lymphomas (CNSLs) are an inhomogeneous group of rare brain tumors consisting of 2 main subtypes: primary and secondary CNSLs. Primary CNSLs account for approx. 7\% of newly diagnosed CNS tumors. ${ }^{1-3}$ Recently, their incidence has increased in immunocompetent patients, though their prevalence is still higher in immunocompromised subjects, such as those with acquired immune deficiency syndrome (AIDS), after organ or bone marrow transplantations, or with inherited immunodeficiency, often due to Epstein-Barr virus (EBV) activation. ${ }^{1-6}$ The majority of primary CNSL tumors are of the highly aggressive diffuse large cell subtypes, usually of B-cell phenotypic origin. ${ }^{4,7,8}$ They are mostly intra-axial lesions located within brain parenchyma, either periventricularly within deep structures, or peripherally along leptomeningeal surfaces. ${ }^{1-3,5}$ They may present as solitary or multiple lesions with usually strong homogeneous enhancement. Lesions found in immunocompromised patients have more commonly multiple locations and tend to enhance less strongly, very often show ring enhancement, as well as features of bleeding and necrosis. ${ }^{3,5,6}$ Secondary CNSLs are metastases to the central nervous system (CNS) from systemic lymphomas, in majority of nodular type; however, metastases to the CNS from extranodal location, including very rare orbital lymphoma, are also possible. ${ }^{1-3,9,10}$ The high aggressiveness of systemic lymphomas, immunodeficiency and extranodal involvement predispose to CNS relapse.,11 The location of secondary CNSLs in brain parenchyma is mainly similar to that of primary CNSLs. Contrary to primary CNSLs, they may also show exclusively extra-axial location within meninges or spine. . $^{3,9,11}$

Since CNSLs may show very variable appearance, a conventional magnetic resonance (MR) examination with contrast injection is not capable of accurately distinguishing them from other intracranial lesions, including tumors, such as gliomas, metastases or even meningiomas. Advanced MR techniques, such as diffusion-weighted imaging (DWI) and perfusion-weighted imaging (PWI), allow for a more detailed analysis of brain tumors and their in vivo differentiation. ${ }^{12-21}$

Diffusion-weighted imaging is a method that evaluates water diffusion in the extracellular space in between the intact cells which constitute a barrier to free movements of water molecules. In tumors, DWI brings information on the tumor cell architecture and the parameter of apparent diffusion coefficient (ADC) is considered as a surrogate marker of tumor cellularity. ${ }^{3,16,19,20}$ Lower values of ADC have been reported in malignant tumors, such as high-grade gliomas, due to their high cellularity rate, and in tumors with high nuclear/cytoplasmatic ratios, such as lymphomas and medulloblastomas. . $^{14,17,15,21}$

On the other hand, PWI is a method that brings information on cerebral physiology at the capillary level (microvasculature). ${ }^{12}$ Among the few PWI techniques, dynamic susceptibility contrast (DSC) magnetic resonance imaging (MRI) is the most often used. Dynamic susceptibility contrast MRI provides maps of cerebral blood volume (CBV) and noninvasive mathematical measurements of relative cerebral blood volume (rCBV).$^{12,22}$ In brain tumors, $\mathrm{rCBV}$ is defined as the ratio between CBV within the tumor and CBV in the white matter of the contralateral hemisphere. The rCBV parameter correlates with tumor vascularity and is increased in tumors with a high rate of pathologic neoangiogenesis. ${ }^{7,12}$ In glial tumors, increased $\mathrm{rCBV}$ ratios indicate increased malignancy, but this rule cannot be applied to extra-axial tumors. There are highly vascular extra-axial tumors with high perfusion, e.g., meningiomas, which are benign in terms of biological behavior. ${ }^{12,15,23}$ Apart from the CBV maps, PWI also provides perfusion curves, which give insights into the dynamics of the first pass of the contrast material through the microvasculature. An analysis of the perfusion curves has been reported to be very important in the evaluation of brain tumors, in some cases even more useful than an analysis of the CBV maps. ${ }^{12,13,17,18,24}$

Accurate identification of CNSLs is crucial from the clinical point of view. Contrary to other malignant tumors, CNSLs do not undergo a surgical management, but are treated with chemotherapy. ${ }^{1,6,18,19}$ At the moment, brain tumors, including CNSLs, still require a biopsy, which is an invasive procedure and can cause unexpected complications.

The aim of our study was a detailed analysis of the conventional MR appearance, as well as diffusion- and perfusion-weighted images of different types of CNSLs in order to establish characteristic imaging patterns, typical for these tumors. To our knowledge, this is the first article presenting diffusion and perfusion results derived from both perfusion maps and curves in various types of CNSLs, both primary and secondary, including immunocompromised and immunodeficient patients. We also discuss the value of different DSC perfusion techniques, with and without pre-bolus, in the accurate diagnosis of CNSLs. ${ }^{25}$

\section{Material and methods}

Our material consisted of 16 brain tumors diagnosed in 12 patients ( 5 men, 7 women) aged 6-84 years with biopsy-proven CNSLs, which were selected from a cohort of 1,060 CNS tumors evaluated with PWI and DWI in our institution in the years 2010-2015. According to the World Health Organization (WHO) system, the evaluated brain tumors were diagnosed either as primary (10 patients) or secondary (2 patients) diffuse large B-cell CNSLs (Table 1). In all patients their clinical history was carefully evaluated. Among primary CNSLs, 8 of 10 patients were found to be immunocompetent, while 2 subjects were immunocompromised (Table 1). Secondary CNSLs were metastases from a systemic nodular lymphoma or extranodular orbital lymphoma (Table 1). 


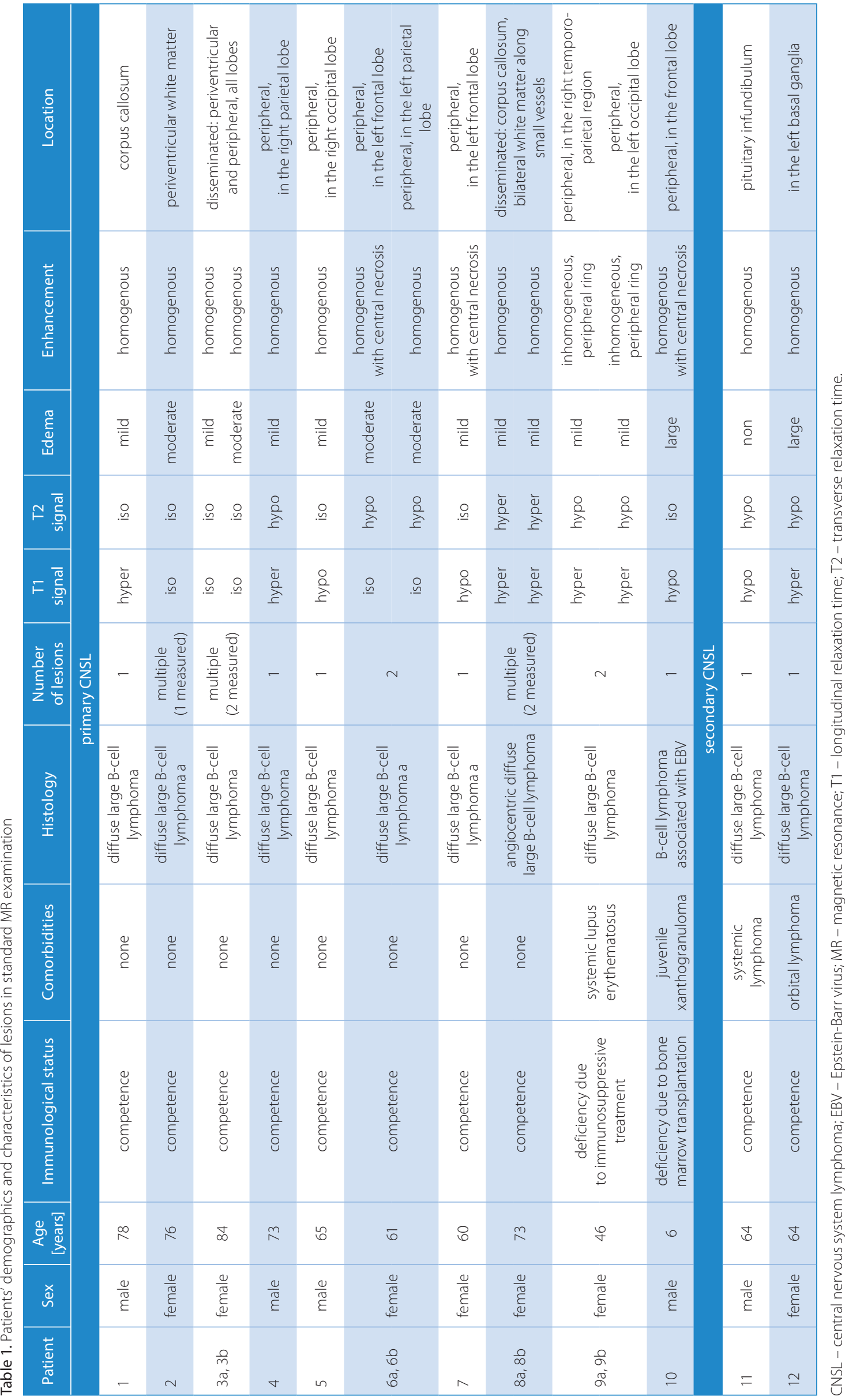


After providing written consent, all patients underwent MR examinations of the brain with contrast injection, including diffusion and perfusion sequences. All procedures were performed in accordance with the Helsinki Human Rights consensus, and the study was approved by the Commission of Bioethics at Wroclaw Medical University.

\section{Data acquisition}

All examinations were performed on a 1.5 T MR scanner (Signa Hdx; GE Medical Systems, Milwaukee, USA), using a 16-channel head-neck-spine (HNS) coil. Before contrast administration, a standard MR examination was carried out, including axial T1 (longitudinal relaxation time)weighted images, axial, coronal and sagittal T2 (transverse relaxation time)-weighted images, as well as axial fluidattenuated inversion recovery (FLAIR) images.

Diffusion-weighted imaging was performed using transverse single-shot echoplanar diffusion-weighted sequence with the following parameters: echo time (TE) - $89.9 \mathrm{~ms}$, repetition time (TR) $-8000 \mathrm{~ms}$, slice thickness $-5 \mathrm{~mm}$, field of view (FOV) $-26 \mathrm{~cm}$, matrix size $-128 \times 128$, number of excitations (NEX) -1.0 , diffusion sensitive gradient $-\mathrm{b}=1000 \mathrm{~s} / \mathrm{mm}^{2}$ in the 3 orthogonal directions, scanning time $-42 \mathrm{~s}$.

Perfusion-weighted imaging was performed with the DSC method using fast echoplanar T2\%-weighted gradient echo sequence with the following parameters: TR $-1.900 \mathrm{~ms}$, $\mathrm{TE}-80 \mathrm{~ms}$, FOV $-30 \mathrm{~cm}$, matrix size $-192 \times 128$, slice thickness $-8 \mathrm{~mm}$ without spacing, NEX -1.0 . A bolus of a $1.0 \mathrm{~mol} / \mathrm{L}$ gadobutrol formula (Gadovist; Bayer Health Care, Leverkusen, Germany) in a dose of $0.1 \mathrm{~mL} / \mathrm{kg}$ of body weight was injected $10 \mathrm{~s}$ after the start of image acquisition via a 20 -gauge catheter placed in the antecubital vein. Contrast was administered with an automatic injector (Medrad; Bayer Medical Care, Indianola, USA) at a rate of $5 \mathrm{~mL} / \mathrm{s}$ and was followed by a saline bolus $(20 \mathrm{~mL}$ at $5 \mathrm{~mL} / \mathrm{s})$. The whole perfusion imaging lasted $1 \mathrm{~min} 26 \mathrm{~s}$, in which sets of images from 13 axial slices were obtained before, during and after contrast injection. After PWI, a postcontrast T1-weighted 3D sequence was performed based on contrast administered earlier in the perfusion examination.

In 2 patients, PWI was performed using the preload leakage correction method. Gadolinium contrast in a dose of $0.05 \mathrm{mmol} / \mathrm{kg}$ was administered as a prebolus $3 \mathrm{~min}$ before the dynamic phase of DSC T2* perfusion; it was then followed by the standard DSC technique as described above.

In the study, 1 patient (Patient 4) underwent 2 types of perfusion examinations - with (4-1) and without (4-2) a preloading bolus in the interval of 4 weeks.

\section{Data postprocessing}

In all cases, the morphological assessment of the lesions was performed on the basis of T1-, T2-weighted and postcontrast T1-weighted images, using visual inspection.

The diffusion- and perfusion-weighted images were postprocessed using Functool software (ADW v. 4.4; GE Medical Systems SCS, Buc, France).

\section{Diffusion-weighted imaging analysis}

The measurements of $\mathrm{ADC}$ for the whole tumor $\left(\mathrm{ADC}_{\mathrm{c}}\right)$ and of minimal $\mathrm{ADC}\left(\mathrm{ADC}_{\mathrm{min}}\right)$ were assessed. The $\mathrm{ADC}_{\mathrm{c}}$ values were obtained by manual outlining of the entire lesion on each slice, and then by calculating the arithmetical means from all measured ADC values. Minimal ADC was measured by placing a small region of interest (ROI) $\left(40-60 \mathrm{~mm}^{2}\right)$ in the location of the lowest value of this parameter on each slice; the lowest value from all the slices was chosen as the tumoral $\mathrm{ADC}_{\mathrm{min}}$. Both $\mathrm{ADC}_{\mathrm{c}}$ and $\mathrm{ADC}_{\text {min }}$ values were normalized to the normal appearing white

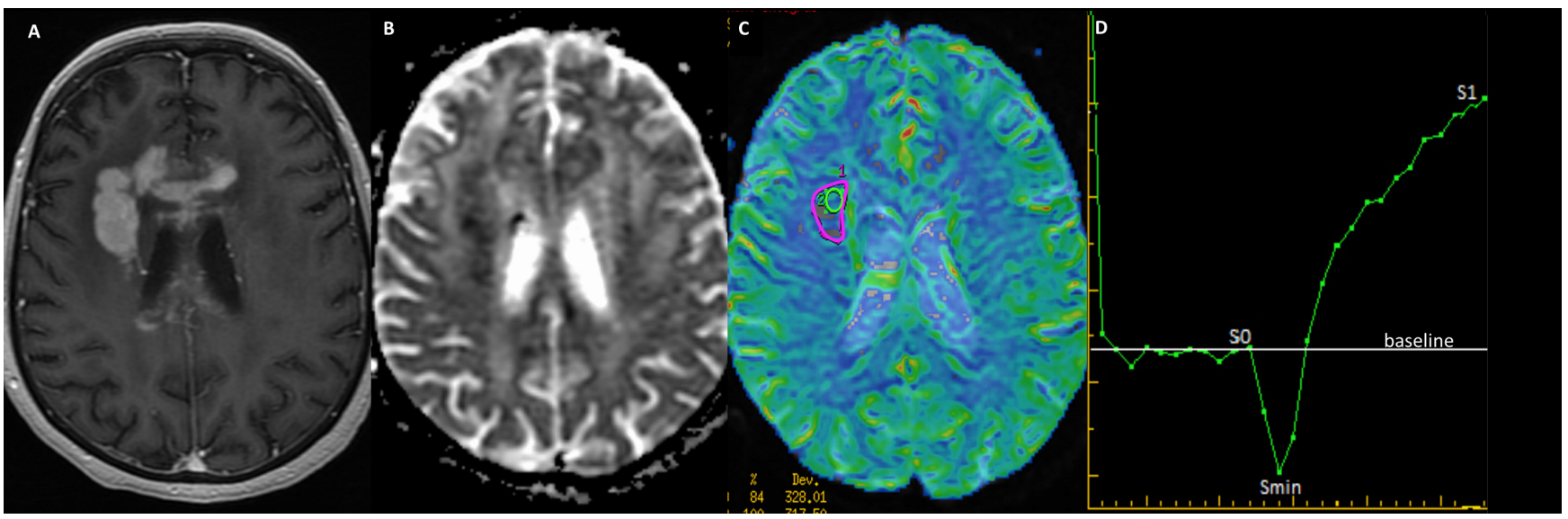

Fig. 1. A typical multifocal primary CNSL located in the basal ganglia and the corpus callosum

A - axial T1-weighted MR image after contrast administration, showing strong enhancement; B - ADC map with restricted diffusion; C - CBV perfusion map with drawn ROIs showing hypoperfusion; D - signal intensity perfusion curve exceeding the baseline level with marked characteristic time points: SO, Smin, S1; ADC - apparent diffusion coefficient; CBV - cerebral blood volume; CNSL - central nervous system lymphoma; MR - magnetic resonance; $\mathrm{ROI}$ - region of interest. 
matter (NAWM) of the contralateral hemisphere in order to obtain the relative values of these parameters (relative $\mathrm{ADC}_{\mathrm{c}}-\mathrm{rADC}_{\mathrm{c}}$; relative $\left.\mathrm{ADC}_{\mathrm{min}}-\mathrm{rADC}_{\mathrm{min}}\right)$.

\section{Perfusion-weighted imaging analysis}

The analysis was based on an evaluation of the CBV parameter on CBV maps, as well as of the values of peak height $(\mathrm{PH})$ and percentage of signal recovery (PSR) derived from perfusion curves. The CBV maps were computed on a pixel-wise basis from the first-pass data as described by Rosen et al. ${ }^{22}$ The measurements of CBV were performed by placing ROIs on the CBV maps fused with post-contrast T1-weighted images in order to accurately assess the tumor core (Fig. 1). The values of PSR and PH were calculated from the perfusion curves based on formulas: $\mathrm{PSR}=(\mathrm{S} 1-\mathrm{Smin}) / \mathrm{PH}, \mathrm{PH}=\mathrm{Smin}-\mathrm{S} 0$, where: $\mathrm{S} 0-$ start of contrast passage, Smin - maximal drop of magnetic susceptibility, S1 - measurement after $24 \mathrm{~s}$ from Smin (Fig. 1). All CBV, PH and PSR values were normalized to values from the NAWM of the contralateral hemisphere in order to obtain relative values of all parameters: $\mathrm{rCBV}$, relative $\mathrm{PH}(\mathrm{rPH})$ and relative PSR (rPSR). ${ }^{23}$

In each tumor, the measurements of mean values of all perfusion parameters for the whole tumor core $\left(\mathrm{rCBV}_{\mathrm{c}}\right.$, $\mathrm{rPH}_{\mathrm{c}}, \mathrm{rPSR}_{\mathrm{c}}$ ) and of maximal values of these parameters $\left(\mathrm{rCBV}_{\max }, \mathrm{rPH}_{\max }, \mathrm{rPSR}_{\max }\right)$ were assessed. The mean values for the whole tumor were obtained by manually outlining the entire lesion on each slice (Fig. 1) and calculating the arithmetical means from all measured values. Maximal values were obtained by placing small ROIs $\left(40-60 \mathrm{~mm}^{2}\right)$ over several hot spots on each slice (Fig. 1). The highest value from all ROIs was chosen as the tumoral maximal value.

\section{Statistical analysis}

Comparisons of diffusion and perfusion values between different subject groups were performed using the t-test with p-values $<0.05$ set as the significance threshold. Diffusion and perfusion parameters were compared between primary and secondary CNSLs, as well as between primary CNSLs in the immunocompetent and immunodeficient patients. We also compared perfusion parameters acquired with and without a preloading bolus.

\section{Results}

\section{Results of standard magnetic resonance examinations}

All CNSLs (both primary and secondary) showed variable $\mathrm{T} 1$ and $\mathrm{T} 2$ appearance ranging from hypo- to hyperintense lesions (Table 1$)$. They were mostly iso- (44\%) or hypointense (44\%) to the white matter on T2-weighted images, with a different range of surrounding edema (Table 1).
All immunocompetent patients showed lesions with strong homogenous enhancement, while in the immunocompromised patients a heterogenous, also ring-like, enhancement pattern was found (Table 1).

In the group of CNSLs (both primary and secondary) there were subjects with single or multiple lesions (Table 1). The location of foci was variable (Table 1). All primary CNSLs were located intra-axially within the brain parenchyma, while secondary lesions were located either intra-axially (basal ganglia) or extra-axially (pituitary infundibulum).

\section{Diffusion results}

All CNSLs showed low mean values of all evaluated diffusion parameters $\left(\mathrm{ADC}_{\mathrm{c}}=0.70 \times 10^{-3}, \mathrm{ADC}_{\min }=0.54 \times 10^{-3}\right)$, which were also lower compared to NAWM $\left(\mathrm{rADC}_{\mathrm{c}}=0.92\right.$ and $\left.\mathrm{rADC}_{\min }=0.71\right)($ Table 2).

Table 2. Results of DWI measurements

\begin{tabular}{|c|c|c|c|c|}
\hline Patients & $\begin{array}{c}\mathrm{ADC}_{c} \\
{\left[\times 10^{-3}\right]}\end{array}$ & $\begin{array}{l}\mathrm{ADC}_{\min } \\
{\left[\times 10^{-3}\right]}\end{array}$ & $\mathrm{rADC}_{\mathrm{c}}$ & $\mathrm{rADC}_{\min }$ \\
\hline \multicolumn{5}{|c|}{ primary CNSLS } \\
\hline \multicolumn{5}{|c|}{ immunocompetent } \\
\hline 1 & 0.57 & 0.42 & 0.77 & 0.67 \\
\hline 2 & 0.76 & 0.47 & 0.89 & 0.55 \\
\hline $3 a$ & 0.81 & 0.56 & 1.05 & 0.72 \\
\hline $3 b$ & 0.81 & 0.67 & 1.05 & 0.86 \\
\hline $4-1$ & 0.69 & 0.53 & 0.88 & 0.73 \\
\hline $4-2$ & 0.69 & 0.62 & 0.88 & 0.78 \\
\hline 5 & 0.59 & 0.52 & 0.77 & 0.67 \\
\hline $6 a$ & 0.65 & 0.57 & 0.89 & 0.78 \\
\hline $6 b$ & 0.96 & 0.73 & 1.33 & 1.01 \\
\hline 7 & 0.65 & 0.54 & 0.93 & 0.77 \\
\hline $8 a$ & 0.58 & 0.47 & 0.77 & 0.62 \\
\hline $8 b$ & 0.65 & 0.56 & 0.86 & 0.74 \\
\hline mean values & 0.70 & 0.55 & 0.93 & 0.74 \\
\hline \multicolumn{5}{|c|}{ immunodeficient } \\
\hline $9 a$ & 0.79 & 0.51 & 1.01 & 0.65 \\
\hline $9 b$ & 0.65 & 0.40 & 0.83 & 0.57 \\
\hline 10 & 0.67 & 0.51 & 0.78 & 0.59 \\
\hline mean values & 0.70 & 0.47 & 0.87 & 0.60 \\
\hline \multicolumn{5}{|c|}{ all primary CNSLs } \\
\hline mean values & 0.70 & 0.54 & 0.92 & 0.71 \\
\hline \multicolumn{5}{|c|}{ secondary CNSLs } \\
\hline 11 & 0.59 & 0.53 & 0.68 & 0.62 \\
\hline 12 & 0.80 & 0.57 & 1.16 & 0.83 \\
\hline mean values & 0.70 & 0.55 & 0.92 & 0.73 \\
\hline \multicolumn{5}{|c|}{ all CNSLs (primary and secondary) } \\
\hline mean values & 0.70 & 0.54 & 0.92 & 0.72 \\
\hline
\end{tabular}

$A D C_{c}$ - apparent diffusion coefficient for the whole tumor;

$A D C_{\text {min }}$ - minimal $A D C ; C N S L$ - central nervous system lymphoma;

$D W I$ - diffusion-weighted imaging; $r A D C_{c}$ - relative $A D C_{c i}$

$r A D C_{\text {min }}$ - relative $A D C_{\text {min }}$. 
Individual analysis of all subjects showed that 5 out of 16 tumors (31\%) revealed $\mathrm{ADC}_{\mathrm{c}}$ values similar or slightly higher compared to NAWM (Patients: 3a, 3b, 6b, 9a, 12) while 11 out of 16 tumors (69\%) showed lower values, indicating restricted diffusion within the entire tumor core (Table 2). When analyzing $\mathrm{ADC}_{\text {min }}$, only 1 subject (Patient $6 \mathrm{~b}$ ) revealed values similar to NAWM. In all other cases, which is $94 \%$ of subjects, the values of $\mathrm{ADC}_{\text {min }}$ were lower than NAWM, which indicated restricted diffusion.

There were no statistically significant differences in all evaluated ADC values between primary and secondary CNSLs, or between immunocompetent and immunodeficient subjects with primary CNSLs (Table 2).

Table 3. Results of PWI measurements

\begin{tabular}{|c|c|c|c|c|c|c|}
\hline Patients & $\mathrm{rCBV}_{\mathrm{c}}$ & $\mathrm{rCBV}_{\max }$ & $\mathrm{rPH}_{\mathrm{c}}$ & $\mathrm{rPH}_{\max }$ & $\mathrm{rPSR}_{\mathrm{c}}$ & $\mathrm{rPSR}_{\max }$ \\
\hline \multicolumn{7}{|c|}{ DSC perfusion without a preload bolus } \\
\hline \multicolumn{7}{|c|}{ primary immunocompetent CNSLs } \\
\hline 1 & 0.72 & 1.02 & 1.20 & 1.59 & 1.55 & 1.80 \\
\hline 2 & 0.40 & 0.83 & 0.99 & 1.30 & 1.95 & 2.11 \\
\hline $3 a$ & 0.45 & 0.52 & 0.74 & 1.10 & 1.48 & 1.54 \\
\hline $3 b$ & 0.91 & 1.85 & 0.83 & 1.14 & 1.47 & 2.43 \\
\hline $4-2$ & 0.81 & 1.11 & 1.22 & 1.41 & 1.50 & 1.85 \\
\hline 5 & 0.53 & 1.70 & 0.97 & 1.76 & 2.49 & 3.95 \\
\hline $6 a$ & 0.31 & 0.44 & 1.05 & 1.29 & 1.95 & 2.10 \\
\hline $6 b$ & 0.53 & 0.87 & 0.80 & 1.85 & 3.24 & 3.25 \\
\hline 7 & 1.41 & 1.65 & 1.43 & 1.89 & 1.31 & 1.51 \\
\hline mean values & 0.67 & 1.11 & 1.03 & 1.48 & 1.88 & 2.28 \\
\hline \multicolumn{7}{|c|}{ primary immunodeficient CNSLs } \\
\hline $9 a$ & 1.12 & 2.17 & 1.49 & 3.01 & 1.49 & 1.67 \\
\hline $9 b$ & 0.93 & 1.04 & 1.32 & 1.70 & 1.23 & 1.29 \\
\hline 10 & 0.43 & 0.79 & 0.84 & 0.86 & 3.78 & 5.83 \\
\hline mean values & 0.82 & 1.33 & 1.22 & 1.86 & 2.17 & 2.93 \\
\hline \multicolumn{7}{|c|}{ all primary CNSLs } \\
\hline mean values & 0.71 & 1.17 & 1.07 & 1.58 & 1.95 & 2.44 \\
\hline \multicolumn{7}{|c|}{ secondary CNSLs } \\
\hline 11 & 1.69 & 2.22 & 0.79 & 1.76 & 2.28 & 2.28 \\
\hline 12 & 1.06 & 1.66 & 0.98 & 1.62 & 2.12 & 2.12 \\
\hline mean values & 1.38 & 1.94 & 0.89 & 1.69 & 2.20 & 2.20 \\
\hline \multicolumn{7}{|c|}{ all CNSLs (primary and secondary) } \\
\hline mean values & 0.80 & 1.27 & 1.05 & 1.59 & 1.99 & 2.41 \\
\hline \multicolumn{7}{|c|}{ DSC perfusion with a preload bolus } \\
\hline $8 a$ & 2.35 & 3.34 & 3.24 & 4.11 & 1.54 & 1.82 \\
\hline $8 b$ & 1.95 & 2.60 & 2.13 & 2.55 & 1.46 & 1.46 \\
\hline $4-1$ & 2.01 & 2.81 & 2.32 & 2.97 & 0.97 & 1.05 \\
\hline mean values & 2.10 & 2.92 & 2.56 & 3.21 & 1.32 & 1.44 \\
\hline \multicolumn{7}{|c|}{ all DSC examinations (with and without a preload bolus) } \\
\hline mean values & 1.04 & 1.58 & 1.31 & 1.81 & 1.87 & 2.24 \\
\hline
\end{tabular}

DSC - dynamic susceptibility contrast; PWI - perfusion-weighted imaging; rCBV ${ }_{c}$ - relative cerebral blood volume for the whole tumor; $\mathrm{rCBV}_{\max }$ - relative maximal $\mathrm{CBV} ; \mathrm{rPH}_{c}$ - relative peak height for the whole tumor; $r \mathrm{PH}_{\max }$ - relative maximal $\mathrm{PH} \mathrm{rPSR}_{\mathrm{c}}$ - relative percentage of signal recovery for the whole tumor; PSR $_{\max }$ - relative maximal PSR.

\section{Perfusion results}

All CNSLs examined using the DSC perfusion method without a preloading bolus showed low mean values of $\mathrm{rCBV}_{c}$, $\mathrm{rCBV}_{\max }, \mathrm{rPH}_{\mathrm{c}}, \mathrm{rPH}_{\max }$, as well as high values of $\mathrm{rPSR}_{\mathrm{c}}$ and $\mathrm{rPSR}_{\max }$, indicating hypoperfusion and overshooting of perfusion curve above the baseline level (Table 3).

Primary CNSLs showed significantly lower $(\mathrm{p}=0.02)$ values of $\mathrm{rCBV}_{\mathrm{c}}$ (mean 0.71; range 0.31-1.41) compared to secondary CNSLs (mean 1.38; range 1.06-1.69). The values of $\mathrm{rCBV}_{\max }$ in secondary CNSLs were slightly higher compared to primary CNSLs, but the difference did not reach the significant level $(\mathrm{p}=0.08)$ (Table 3$)$. The values of $\mathrm{rCBV}_{\max }$ in primary CNSLs ranged from 0.44 to 2.17 , while in secondary CNSLs from 1.66 to 2.22 . Only 3 out of 14 tumors (21.4\%) showed values of $\mathrm{rCBV}_{\max }$ higher than 1.75 , but not exceeding 2.22. There were no significant differences in the values of perfusion parameters derived from perfusion curves between primary and secondary CNSLs.

When immunocompetent and immunodeficient patients were compared with primary CNCLs, no differences were found in the values of all perfusion parameters between these 2 groups.

Compared to DSC without a preload bolus, primary CNSLs, which were examined using a preload bolus, revealed statistically higher $(\mathrm{p}<0.001)$ mean values of $\mathrm{rCBV}_{\mathrm{c}}$ (mean 2.10; range 1.95-2.35), $\mathrm{rCBV}_{\max }$ (mean 2.92; range 2.6-3.34), $\mathrm{rPH}_{\mathrm{c}}$ (mean 2.56), and $\mathrm{rPH}_{\max }$ (mean 3.33), as well as significantly lower rPSR measurements (Table 3). Patient 4 examined with both perfusion techniques also showed significantly different results of all perfusion parameters with higher rCBV and $\mathrm{rPH}$ values as well as lower rPSR values in the DSC technique with a use of a preloading bolus (4-1) compared to the follow-up examination without a preload (4-2) (Table 3).

\section{Discussion}

Our study based on the group of 16 CNSLs tumors showed a great variety of radiological appearances on conventional MR sequences, which is typical for these tumors. We observed a wide 


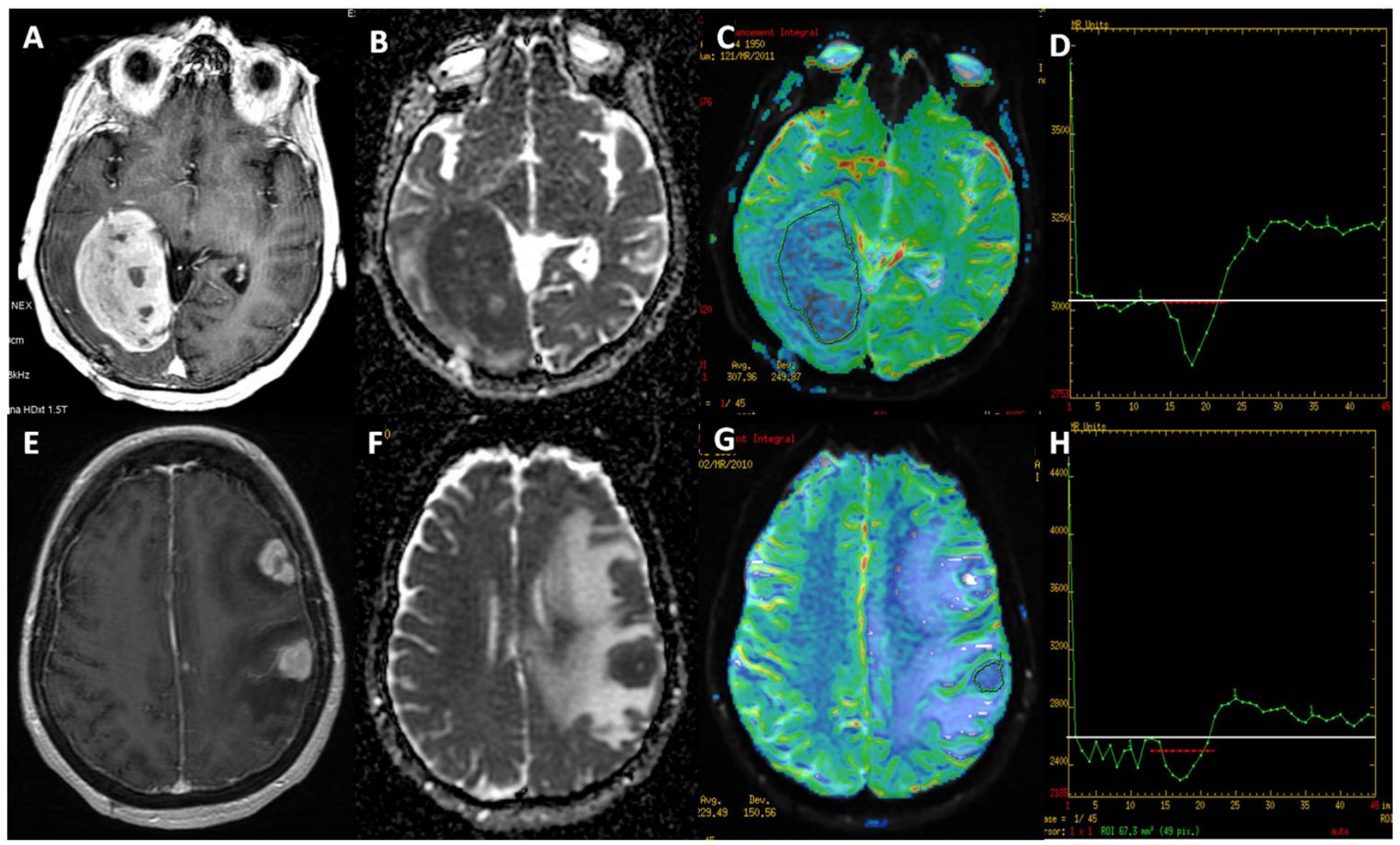

Fig. 2. Primary CNSLs (in immunocompetent patients) mimicking other tumors such as meningioma (A-D) and metastases (E-H)

All CNSLs show strong homogeneous enhancement on postcontrast T1-weighted images (A, E), restricted diffusion on ADC maps (B, F), low perfusion on CBV maps (C, G) and a typical shape of the perfusion curves above the baseline $(D, H)$.

ADC - apparent diffusion coefficient; CBV - cerebral blood volume; CNSL - central nervous system lymphoma.

range of signal changes on T1- and T2-weighted images, different patterns of contrast enhancement, as well as different numbers and locations of tumoral foci.

$\mathrm{T} 2$ hypointensity is reported to be one of the very characteristic features of CNSLs, attributed to their high nuclear/cytoplasmatic ratio. ${ }^{1-3,14}$ In the group of our subjects, we found low T2 signal in 44\% of all CNSLs, T2 isointensity also in $44 \%$ of CNSLs, while high T2 signal only in $12 \%$ of all subjects. T2 hypointensity was found in all groups of evaluated primary and secondary lymphomas, as well as in immunocompromised and immunocompetent subjects. In our study, all subjects with CNSLs with competent immune system revealed solid and homogenous contrast enhancement (84.6\%), which was also reported in the literature. ${ }^{1-3,14}$ On the other hand, immunocompromised individuals showed inhomogeneous enhancement, including a peripheral ring-like pattern. This inhomogeneity is found to be typical and is explained by lower focal cellularity, resulting from tumor necrosis in patients with depressed immunity. ${ }^{1-3,14,16}$ The number of lesions in each patient varied from a single tumor found in all subject groups to multiple foci characteristic for primary CNSLs in immunocompetent patients. Almost all evaluated tumors showed an intra-axial location, either peripherally, or within deep structures, except for 1 case of secondary lymphoma within pituitary infundibulum, which is also consistent with literature reports. ${ }^{2,3}$
To summarize the findings of standard MR examinations, in our study, primary CNSLs in immunocompetent patients were intra-axial lesions with various T2 signal, as well as strong and solid enhancement, with a tendency to appear in multiple foci. On the other hand, primary CNSLs in immunodeficient patients appeared as T2 hypoor isointense lesions with inhomogeneous enhancement, including a ring-like pattern with peripheral intra-axial location, while secondary CNSLs were lesions with very similar MR appearance to primary CNSLs in immunocompetent subjects, apart from their tendency to appear in an extra-axial location.

As presented above, CNSLs in our study were a highly variable group of tumors in standard MR imaging, mimicking other focal brain lesions. All cases of single lesions required differentiation from other brain tumors, including gliomas and even meningiomas due to their tendency to contact with meningeal surfaces (Fig. 2 A-D). Multiple homogenous masses with diffuse surrounding edema were highly suggestive of metastases (Fig. $2 \mathrm{E}-\mathrm{H}$ ), while multiple disseminated lesions strongly mimicked inflammatory changes or angitis in 1 case (Patient 8).

As mentioned by Haldorsen et al. and also confirmed by an analysis of our material, standard MR imaging is not sufficient to unequivocally differentiate CNSLs from other brain lesions, thus newer advanced imaging techniques 
should be added to aid the accurate diagnosis of these tumors. ${ }^{3}$ Several techniques have been reported to improve the differentiation of CNSLs, including DWI, PWI, MR spectroscopy, and diffusion tensor imaging (DTI). ${ }^{12,16,19,20}$ To our knowledge there are still no reports on a detailed analysis of both DWI and PWI in various CNSLs, including primary and secondary disease, as well as various immune status of evaluated patients.

Diffusion-weighted imaging is an imaging technique which may provide information regarding tumor cellularity and biology, based on the fact that cells constitute a barrier to water diffusion. Highly cellular tumors such as lymphomas tend to demonstrate a restriction of water diffusion, and thus decreased values of the ADC parameter. ${ }^{16,18-20}$ In our study, an analysis of DWI results showed low mean values of $\mathrm{ADC}_{\mathrm{c}}$ and $\mathrm{ADC}_{\text {min }}$ in the group of all evaluated subjects, which is consistent with previous reports. ${ }^{16,18-20}$ We also looked at individual subjects and revealed that in $69 \%$ of all evaluated lymphomas, the $\mathrm{ADC}_{\mathrm{c}}$ values were lower than in NAWM, indicating homogenously restricted diffusion within the entire tumor load, and when searching for areas of the lowest ADC values $\left(A D C_{m i n}\right), 94 \%$ of evaluated lesions showed small regions of restricted diffusion.

In our study, we also did not find any significant differences in all evaluated ADC values between primary and secondary, or between immunocompetent and immunodeficient subjects with primary CNSLs, which indicates that restricted diffusion is a consistent DWI finding in all CNSLs. We did not find any other DWI studies comparing primary and secondary lymphomas in the literature. On the other hand, Zacharia et al. evaluated the ADC parameters in immunocompetent and immunodeficient primary CNSLs but without detailed comparisons between these 2 groups of patients. ${ }^{16}$ They focused on comparisons between lesions with homogenous, inhomogeneous and ring-like enhancement, reporting restricted diffusion in all tumors except for 2 ring-enhancing lesions, which led to an incorrect diagnosis of the infection. It has to be stressed that in our study of ring-enhancing lymphomas, all DWI parameters were obtained from the periphery of these lesions and in all cases we found large areas of restricted diffusion. It is crucial to identify correctly areas of restricted diffusion in inhomogeneously enhancing lymphomas, since this is a characteristic feature of these tumors, which can be used to differentiate them from others, such as gliomas or metastases, which show higher ADC values or only small areas of restricted diffusion consistent with the most malignant parts of these tumors. ${ }^{6,14,17,18,21}$

In the next part of our study, we also evaluated the results of perfusion examinations. In the majority of cases, we used the DSC perfusion method without a preload, which is the most often performed perfusion technique in the evaluation of focal brain lesions. Relative CBV is the most important perfusion parameter in the evaluation of brain tumors, which correlates with their regional vascularity and is increased in tumors with a high rate of pathologic neoangiogenesis. ${ }^{12}$ Since most brain tumors are in some parts inhomogeneous, the parameter of $\mathrm{rCBV}_{\max }$ is the most important and commonly used in everyday practice as the one reflecting the most malignant areas within a tumor core (the so-called hot spots). In glial tumors, increased rCBV ratios indicate increased malignancy, but this rule cannot be applied to other tumors, for example meningiomas, which are highly perfused lesions, but benign in terms of biological behavior, or lymphomas, which are malignant lesions with relatively low perfusion values. Low values of $\mathrm{rCBV}$ in lymphomas can be explained by the histopathological appearance of these tumors - their high cellularity, complete absence of neoangiogenesis and angiocentric growth pattern. ${ }^{12}$ In our study, similarly to previous reports, low mean values of $\mathrm{rCBV}$ (both $\mathrm{rCBV}_{\mathrm{c}}$ and $\mathrm{rCBV}_{\max }$ ) ranging from 0.44 to 2.22 were found within all evaluated lymphomas. ${ }^{3,12-14}$ Several studies have demonstrated that glioblastomas (GBMs) and metastases may reach very high $\mathrm{rCBV}_{\max }$ values of 3.0 or even 10.0, mainly due to a high concentration of microvessels, ${ }^{12,24}$ and rCBV of 1.75 has been set as the threshold value differentiating low-grade and highgrade gliomas. ${ }^{26,27}$ It has to be pointed out that $79 \%$ of all CNSLs in our study showed $\mathrm{rCBV}_{\max }$ values $<1.75$, similarly to low grade gliomas, and $21 \%$ revealed $\mathrm{rCBV}_{\max }$ values $>1.75$, but not exceeding 2.22, which is very unusual for high-grade gliomas, metastases or meningiomas. Though lymphomas may strongly mimic high-grade gliomas, metastases or even meningiomas in their conventional MR appearance, they show low perfusion values similarly to low-grade gliomas, which may be a very helpful feature in the correct differential diagnosis of these lesions.

Subsequently, we also evaluated the perfusion curves of CNSLs. Low rPH and high rPSR values were observed in the majority of cases. High $\mathrm{rPSR}_{\max }$ in lymphomas is consistent with an overshooting of the signal intensity curve above the baseline level, which was observed in 100\% of our cases. The rPSR ratio was reported by Mangla et al. to be the most sensitive and specific feature in the differentiation of lymphomas from GBMs and metastases. ${ }^{12}$ Furthermore, there are other reports of significantly reduced PSR in metastases and GBMs compared to CNSLs, reflected in the perfusion curve not crossing the baseline. ${ }^{12,14}$

Since hypoperfusion in lymphomas can be explained by hypovascularization and the absence of neoangiogenesis, the exact explanation of the signal intensity curve returning above the baseline level is difficult and not fully understood. ${ }^{12,14}$ It is probably due to gadolinium extravasation into the interstitial space and complex T1 and T2 effects, which can alter the shape of the perfusion curve. T2 effects lead to lower signal intensity recovery, while T1 effects cause higher signal intensity recovery. ${ }^{12,28}$ In lymphomas, T1 effects, probably due to an extensive accumulation of contrast material in the interstitial space, dominate over T2 effects and cause the characteristic overshooting from the baseline. ${ }^{12,28,29}$ However, so far, no definite explanation has been 


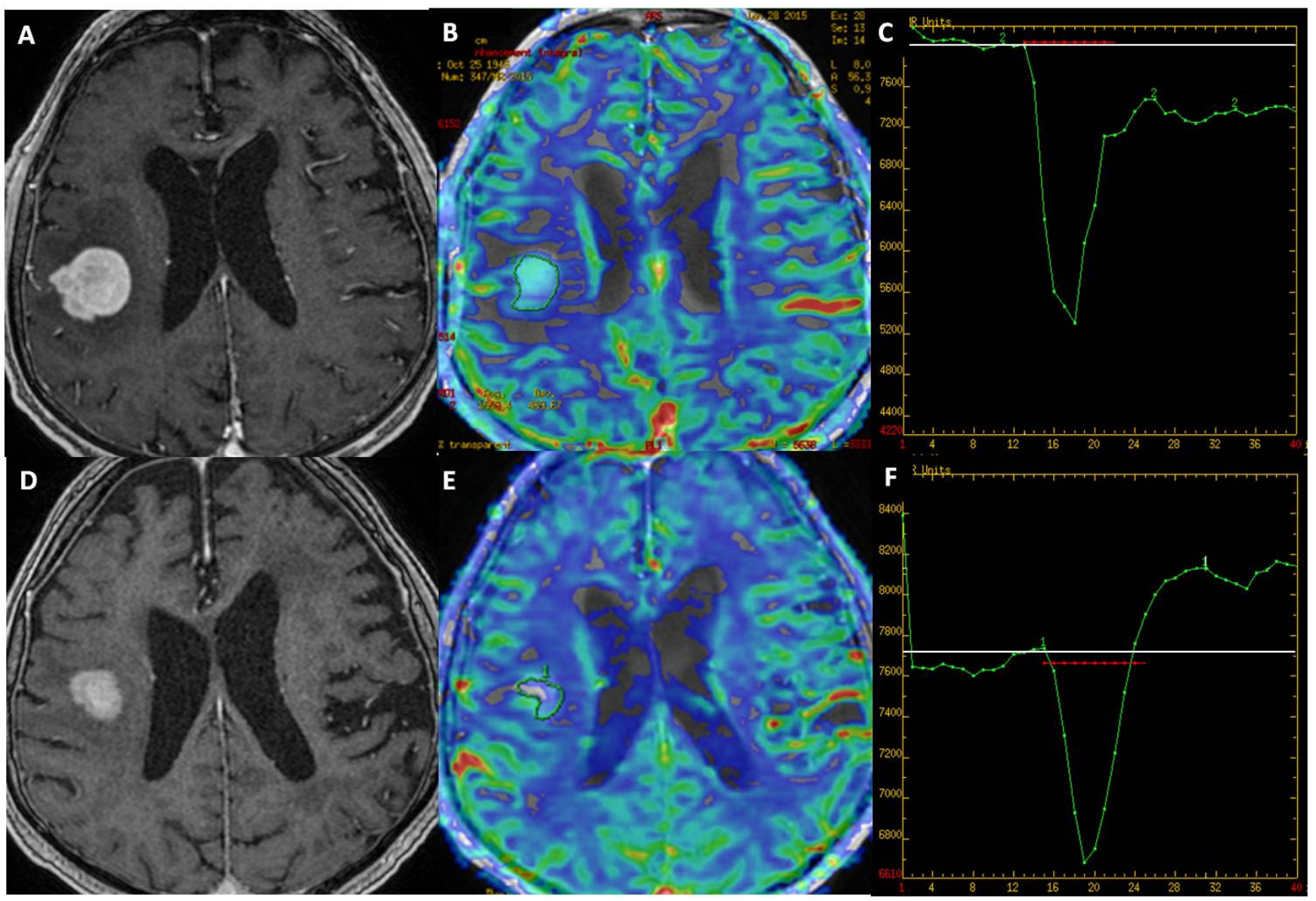

Fig. 3. A patient with a single CNSL lesion in the right parietal lobe examined with 2 DSC perfusion techniques with (A-C) and without (D-F) a preloading bolus

Axial T1-weighted MR images (A, D) after contrast administration, showing strong enhancement. The CBV perfusion map with a preload (B) shows hyperperfusion compared to hypoperfusion seen on the CBV map without a preload (E). The perfusion curve after a preload (C) exceeds the baseline compared to the perfusion curve without a preload, which does not reach the baseline (F).

CBV - cerebral blood volume; CNSL - central nervous system lymphoma; DSC - dynamic susceptibility contrast; MR - magnetic resonance.

given for overshooting in lymphomas in DSC perfusion without a preload. This phenomenon is probably caused by several factors and a complex interplay between them. ${ }^{12}$

Moreover, in our study we also compared all perfusion parameters between primary and secondary CNSLs, as well as between immunocompromised and immunocompetent patients. Secondary CNSLs revealed significantly higher $\mathrm{rCBV}_{\mathrm{c}}$ values compared to primary CNSLs, but still lower than 1.75. There were no other significant differences in any other perfusion parameters derived from CBV maps or perfusion curves among all evaluated patients subgroups. To our knowledge there are no reports in the literature that compare perfusion parameters in various lymphoma subgroups.

The last part of our investigation was to compare the results of 2 different perfusion techniques - with and without a preload bolus of contrast. The perfusion results after a preload indicated hyperperfusion of the evaluated lymphomas (high rCBV and rPH values) and showed the perfusion curves with only a partial return to the baseline level (low rPSR values). These results differed significantly from the values obtained in the perfusion technique without a preload.
In a few previous studies their authors have also analyzed CNSLs in PWI with a preload. ${ }^{15,25,30}$ Preload dosing techniques have been proposed to minimize and correct for T1-weighted leakage due to blood-brain barrier, as well as $\mathrm{T} 2$ - and/or T2*-weighted imaging residual effects characteristic for DSC perfusion techniques without a preload. These techniques are useful in differentiating between post-treatment radiation injury and recurrence of highgrade gliomas, where the blood-brain barrier disruption is significant and can lead to an underestimation of $\mathrm{rCBV}$ measurements. ${ }^{7,9,23}$ On the other hand, a preload in CNSLs elevates their CBV values and effaces the characteristic perfusion curve, making them more similar to perfusion characteristics of high-grade gliomas, metastases or meningiomas. Therefore, in our opinion this technique brings a disadvantage in the case of lymphomas, because it makes their differentiation from other tumors impossible. ${ }^{15,25,30}$

A good practical example illustrating the differences between DSC methods with and without a preload is the case of Patient 4, who was examined with both techniques. The initial examination with a preload resulted in high rCBV values with $\mathrm{rCBV}_{\max }$ reaching 2.81 and a wrong diagnosis 
of a metastasis (Fig. $3 \mathrm{~A}-\mathrm{C}$ ). The follow-up examination without a preload presented typical perfusion characteristics of CNSLs, such as hypoperfusion and the perfusion curve returning above the baseline level, which enabled the correct diagnosis of lymphoma confirmed later in biopsy (Fig. 3 D-F).

\section{Conclusions}

Despite their various appearances in conventional MR examinations, CNSLs (both primary or secondary and in patients with different immunological status) show very typical patterns in DWI and PWI without a preload bolus, such as diffusion restriction and hypoperfusion with the signal intensity curves returning above the baseline, respectively. These features enable us to differentiate CNSLs from other brain tumors, such as high-grade gliomas, metastases or meningiomas. In our opinion, advanced MR techniques such as DWI and PWI without a preload, as methods easy to perform and interpret, should be routinely incorporated in the initial workup of all brain tumors.

\section{References}

1. Osborn AG, Salzman KL, Barkovich AJ. Diagnostic imaging: Brain. $2^{\text {nd }}$ ed. Salt Lake City, UT: Amirsys Publishing; 2010.

2. Haldorsen IS, Krossnes BK, Aarseth JH, et al. Increasing incidence and continued dismal outcome of primary central nervous system lymphoma in Norway 1989-2003: Time trends in a 15-year national survey. Cancer. 2007;110(8):1803-1814.

3. Haldorsen IS, Espeland A, Larsson EM. Central nervous system lymphoma: Characteristic findings on traditional and advanced imaging. Am J Neuroradiol. 2011;32(6):984-992.

4. Miller DC, Hochberg FH, Harris NL, Gruber ML, Louis DN, Cohen H. Pathology with clinical correlations of primary central nervous system non-Hodgkin's lymphoma. The Massachusetts General Hospital experience 1958-1989. Cancer. 1994;74(4):1383-1397.

5. Bhagavathi S, Wilson JD. Primary central nervous system lymphoma. Arch Pathol Lab Med. 2008;132(11):1830-1834.

6. Neska-Matuszewska M, Zimny A, Kałwak K, Sąsiadek MJ. Central nervous system lymphoma in a 3-year-old male suffering from a severe juvenile xanthogranuloma - The usefulness of perfusion weighted imaging and diffusion weighted imaging in the diagnostics of pediatric brain tumors. Pol J Radiol. 2015;80:31-35.

7. Hill QA, Owen RG. CNS prophylaxis in lymphoma: Who to target and what therapy to use. Blood Rev. 2006;20(6):319-332.

8. Krogh-Jensen $M$, Johansen $P, D^{\prime}$ Amore F. Primary central nervous system lymphoma in immunocompetent individuals: Histology, Epstein-Barr virus genome, Ki-67 proliferation index, p53 and bcl-2 gene expression. Leuk Lymphoma. 1998;30(1-2):131-142.

9. Senocak E, Oguz KK, Ozgen B, et al. Parenchymal lymphoma of the brain on initial MR imaging: A comparative study between primary and secondary brain lymphoma. Eur JRadiol. 2011;79(2):288-294.

10. Ferreri AJ, Dolcetti R, Du MQ, et al. Ocular adnexal MALT lymphoma: An intriguing model for antigen-driven lymphomagenesis and microbial-targeted therapy. Ann Oncol. 2008;19(5):835-846.

11. Cavaliere R, Petroni G, Lopes MB, Schiff D; International primary central nervous system lymphoma collaborative group. Primary central nervous system posttransplantation lymphoproliferative disorder: An international primary central nervous system lymphoma collaborative group report. Cancer. 2010;116(4):863-870.

12. Mangla R, Kolar B, Zhu T, Zhonga J, Almasta J, Ekholma S. Percentage of signal recovery derived from MR dynamic susceptibility contrast imaging is useful to differentiate common enhancing malignant lesions of the brain. Am J Neuroradiol. 2011;32(6):1004-1010.
13. Cha S, Lupo JM, Chen MH, et al. Differentiation of glioblastoma multiforme and single brain metastasis by peak height and percentage of signal intensity recovery derived from dynamic susceptibility-weighted contrast-enhanced perfusion MR imaging. Am J Neuroradiol. 2007;28(6):1078-1084.

14. Rizzo L, Crasto SG, Moruno PG, et al. Role of diffusion- and perfusionweighted MR imaging for brain tumour characterisation. RadiolMed. 2009;114(4):645-646.

15. Sugahara T, Korogi $Y$, Tomiguchi $S$, et al. Posttherapeutic intraaxial brain tumor: The value of perfusion-sensitive contrast-enhanced MR imaging for differentiating tumor recurrence from nonneoplastic contrast-enhancing tissue. Am J Neuroradiol. 2000;21(5):901-909.

16. Zacharia TT, Law M, Naidich TP, Leeds NE. Central nervous system lymphoma characterization by diffusion-weighted imaging and MR spectroscopy. J Neuroimaging. 2008;18(4):411-417.

17. Calli C, Kitis O, Yunten N, Yurtseven T, Islekel S, Akalin T. Perfusion and diffusion MR imaging in enhancing malignant cerebral tumors. Eur J Radiol. 2006;58(3):394-403.

18. Provenzale JM, Mukundan S, Barboriak DP. Diffusion-weighted and perfusion MR imaging for brain tumor characterization and assessment of treatment response. Radiol. 2006;239(3):632-649.

19. Barajas RF, Rubenstein JL, Chang JS, Hwang J, Cha S. Diffusionweighted MR imaging derived apparent diffusion coefficient is predictive of clinical outcome in primary central nervous system lymphomas. Am J Neuroradiol. 2012;31(1):60-66.

20. Schroeder PC, Post MJ, Oschatz E, Stadler A, Bruce-Gregorios J, Thurnher MM. Analysis of the utility of diffusion-weighted MRI and apparent diffusion coefficient values in distinguishing central nervous system toxoplasmosis from lymphoma. Neuroradiol. 2006;48(10):715-720.

21. Guo AC, Cummings TJ, Dash RC, Provenzale JM. Lymphomas and high-grade astrocytomas: Comparison of water diffusibility and histologic characteristics. Radiol. 2002;224(1):177-183.

22. Rosen B, Belliveau J, Vevea J, Brady TJ. Perfusion imaging with NMR contrast agents. Magn Reson Med. 1990;14(2):249-265.

23. Zimny A, Sąsiadek M. Contribution of perfusion-weighted magnetic resonance imaging in the differentiation of meningiomas and other extra-axial tumors: Case reports and literature review. J Neurooncol. 2011;103(3):777-783.

24. Cha S, Knopp EA, Johnson G, Wetzel SG, Litt AW, Zagzag D. Intracranial mass lesions: Dynamic contrast-enhanced susceptibility-weighted echo-planar perfusion MR imaging. Radiol. 2002;223(1):11-29.

25. Hu LS, Baxter LC, Pinnaduwage DS, et al. Optimized preload leakagecorrection methods to improve the diagnostic accuracy of dynamic susceptibility-weighted contrast-enhanced perfusion MR imaging in posttreatment gliomas. Am J Nuroradiol. 2010;31(1):40-48.

26. Essig M, Anzalone N, Combs SE, et al. MR imaging of neoplastic central nervous system lesions: Review and recommendations for current practice. Am J Neuroradiol. 2012;33(5):803-817.

27. Law M, Oh S, Johnson G, et al. Perfusion magnetic resonance imaging predicts patients outcome as an adjunct to histopathology: A second reference standard in the surgical and nonsurgical treatment of lowgrade gliomas. Neurosurgery. 2006;58(6):1099-1107.

28. Paulson ES, Schmainda KM. Comparison of dynamic susceptibility-weighted contrast-enhanced MR methods: Recommendations for measuring relative cerebral blood volume in brain tumors. Radiol. 2008;249(2):601-613.

29. Runge VM, Kirsch JE, Wells JW, Dunworth JN, Hilaire L, Woolfolk CE. Repeat cerebral blood volume assessment with first-pass MR imaging. J Magn Reson Imaging. 1994;4(3):457-461.

30. Toh CH, Wei KC, Chang CN, Ng SH, Wong HF. Differentiation of primary central nervous system lymphomas and glioblastomas: Comparisons of diagnostic performance of dynamic susceptibility contrastenhanced perfusion MR imaging without and with contrast-leakage correction. Am J Neuroradiol. 2013;34(6):1145-1149. 